\title{
Un modelo microeconómico estocástico del comportamiento del migrante mexicano en Estados Unidos
}

\author{
Rosa María Domínguez Gijón* \\ Francisco Venegas Martínez**
}

En este artículo se desarrolla un modelo estocástico que explica el comportamiento de un migrante en Estados Unidos. En el modelo propuesto el agente tiene un consumo de subsistencia sujeto a dos restricciones: en la primera se considera un ingreso incierto junto con un ahorro y en la segunda su rutina de consumo. Por medio del modelo propuesto se determinan las proporciones óptimas del ingreso que el individuo obtiene por su actividad laboral y el ahorro cuando hay un excedente, asi como la estrategia óptima de consumo futuro. Asimismo, mediante un modelo VAR se muestra evidencia empírica que confirma los resultados de la propuesta: se utilizan datos de consumo y salario del individuo y variables relevantes asociadas con los migrantes desde 1980 hasta 2011 valiéndose de un análisis de causalidad de Granger, funciones de impulso-respuesta y descomposición de la varianza.

Palabras clave: ingreso incierto, consumo de subsistencia, formación de hábitos, programación dinámica estocástica, migrantes.

Fecha de recepción: 24 de junio de 2013.

Fecha de aceptación: 16 de diciembre de 2013.

\section{A Microeconomic Stochastic Model of the Behavior of Mexican Migrants in the U.S.}

This paper develops a stochastic model that explains the behavior of an immigrant in the United States. In the proposed model, the agent has a subsistence consumption subject to two restrictions: the first considers an irregular income together with saving, while the second considers his consumption routine. The proposed model is used to determine the optimal proportions of income the individual obtains from his work and saving when there is a surplus, and the optimal strategy for future consumption. Thus, a VAR model provides empirical evidence confirming the results of the proposal: data on the consump-

* Candidata a doctora en Ciencias Económicas, Escuela Superior de Economía, IPN. Dirección postal: Plan de Agua Prieta 66, Plutarco Elías Calles, 11350, México, D.F., México. Correo electrónico: <rossy13pink@hotmail.com>.

** Profesor de tiempo completo en el posgrado de la Escuela Superior de Economía, IPN. Dirección postal: Plan de Agua Prieta 66, Plutarco Elías Calles, 11350, México, D.F., México. Correo electrónico:<fvenegas1111@yahoo.com.mx>.

ESTUDIOS DEMOGRÁFICOS Y URBANOS, VOL. 30, NÚM. 1 (88), 2015, 45-75 
tion and salary of the individual are used, together with relevant variables associated with migrants from 1980 to 2011, using a Granger causality analysis, impulse response functions and variance decomposition.

Key words: uncertain income, subsistence consumption, habit formation, stochastic dynamic programming, migrants.

\section{Introducción}

La migración es un fenómeno demográfico que se ha registrado en todos los periodos históricos en formas y grados diversos, como lo muestran entre otros los estudios de Lozano (1998 y 1999), Garavito y Torres (2004), Tuirán (2006) y Heredia (2011), y suele definirse como el cambio de residencia habitual mediante el desplazamiento de una unidad geográfica a otra (país, entidad federativa). ${ }^{1}$

El crecimiento del empleo en México ha sido insuficiente para atender a un grupo de individuos excedente que no ha logrado insertarse en el empleo formal, ${ }^{2}$ en donde el salario es de forma fija. Muchos de estos individuos han tenido que crear su propia fuente de empleo en actividades tales como el comercio en la economía informal, ${ }^{3} \mathrm{o}$ bien han tenido que emigrar hacia Estados Unidos atraídos por la demanda de mano de obra mexicana ${ }^{4}$ en los sectores agrícola y de servicios, convirtiéndose así en migrantes cuyo incentivo es un diferencial salarial entre México y Estados Unidos (al tipo de cambio de 2012 que muestra la gráfica 1), el cual les permitirá mantenerse a sí mismos y a sus familias, decidiendo las horas que deben trabajar para cubrir su consumo diario.

Según el U.S. Census Bureau la migración hacia Estados Unidos fue de 31798258 mexicanos en 2010 que representó 63\% del total de migrantes en ese país, quienes se concentraron principalmente en los estados de California y Texas. Por otro lado, de acuerdo con el Conapo, los estados con mayor migración son Zacatecas, Jalisco, Michoacán y Oaxaca, aunque este fenómeno ya se presenta en toda la república.

1 INEGI, 2010, glosario.

2 Aunque muchos individuos simplemente no desean insertarse en el sector formal y migrar a Estados Unidos.

${ }_{3}^{3}$ De acuerdo con el INEGI el sector informal aporta alrededor del 13\% del PIB y da ocupación a más de 14 millones de personas.

${ }^{4}$ La migración de origen mexicano es la de mayor crecimiento entre la latina, y en el estado de California es la primera minoría. 


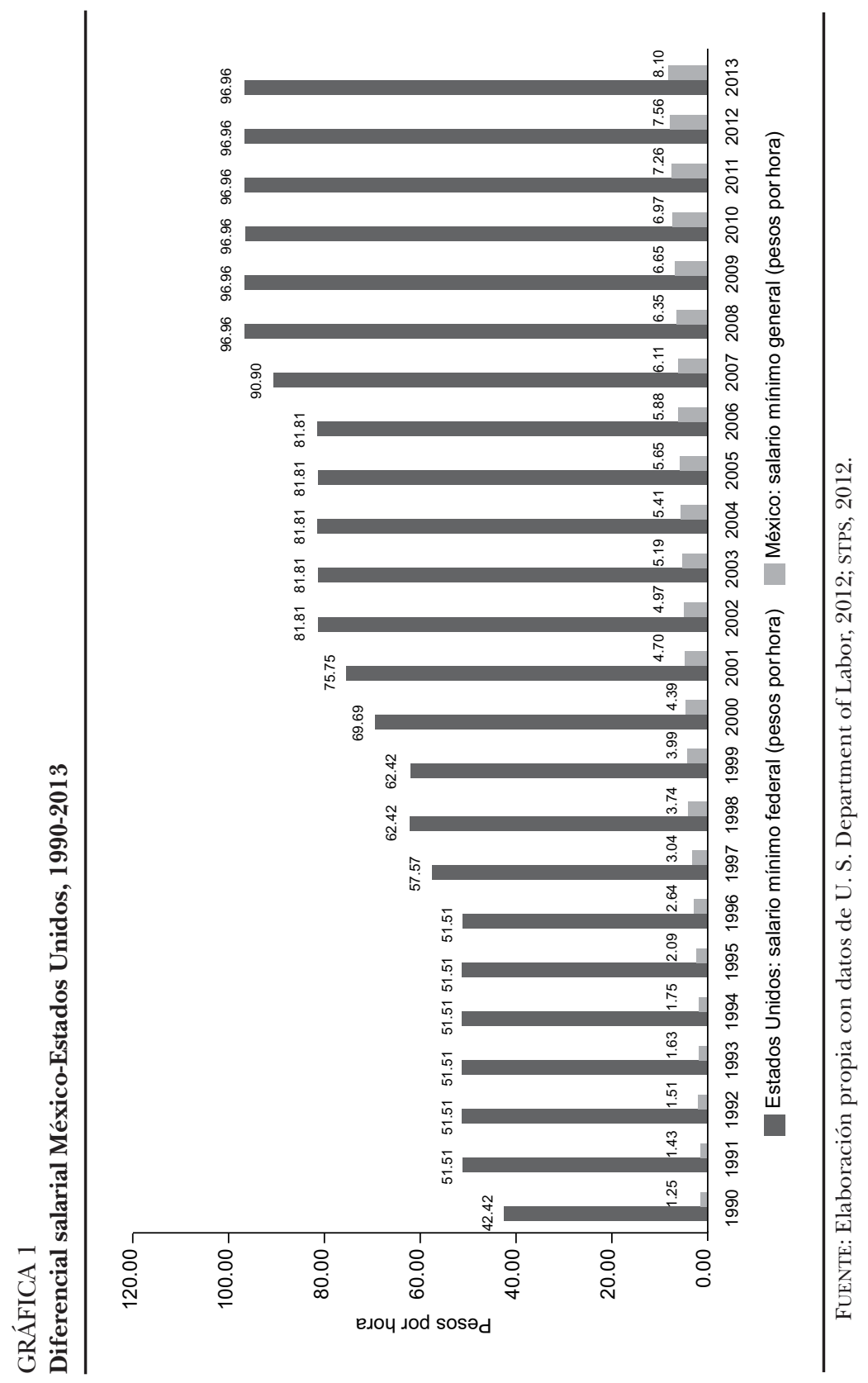


El migrante recibe en promedio 22500 pesos mensuales por una jornada de ocho horas diarias, lo que equivale a casi 15 veces el salario mínimo de México. En la actualidad el poder adquisitivo de dicho salario se ha reducido considerablemente.

El envío de remesas depende de que el migrante mantenga su fuente de trabajo. Éstas se utilizan principalmente para cubrir las necesidades más esenciales, como alimentos, vivienda y acceso a los servicios públicos (salud y educación), como se aprecia en los estudios de Garavito y Torres (2004), Castillo (2001) y Santiago (2001), y en los de Lozano (1998 y 1999), quien formuló un algoritmo para calcular las remesas. En la gráfica 2 se observa un incremento considerable de envíos de remesas a México de 1990 a 2007, mientras que la crisis de 2009 redujo los flujos de remesas, lo que ha comprometido parte del ingreso de muchos hogares mexicanos.

En esta investigación se desarrolla un modelo que explica el comportamiento de un migrante que reside en Estados Unidos ${ }^{5}$ y que toma decisiones sobre la cantidad de horas que dedicará a la actividad laboral que ha de proporcionarle un ingreso incierto y un consumo de subsistencia, ya que con miras a enviar la mayor cantidad posible de dinero a sus familiares mantiene la mínima cantidad necesaria para su sustento. En cuanto al consumo de subsistencia, Constantinides (1990) introdujo el concepto de formación de hábitos de consumo (o rutina de consumo), el cual se utilizará en el estudio del comportamiento de este agente. De igual forma Constantinides estudia el problema de las decisiones de consumo y de portafolio en presencia de una restricción de formación de hábitos en la que el individuo tiene en cuenta el efecto del consumo pasado sobre su consumo futuro en un modelo de equilibrio parcial. Asimismo, la noción de formación de hábitos ha sido también utilizada por Hicks (1965), Pollak (1970), Ryder y Heal (1973), Sundaresan (1989), Andrew (1990), Detemple y Zapatero (1991), Chapman (1998), Campbell y Cochrane (1999), Zheng y Xu (2003) y Márquez (2006), entre muchos otros.

Por otro lado, el presente trabajo amplía varias investigaciones sobre el problema del ingreso incierto; por ejemplo, se generalizan las investigaciones de Merton (1969 y 1971) sobre las políticas óptimas

\footnotetext{
${ }^{5}$ Cabe mencionar que también puede ser aplicable a cualquier individuo representativo que cumpla con ciertas características y que tome decisiones sobre la cantidad de horas que dedicará a la actividad laboral.
} 


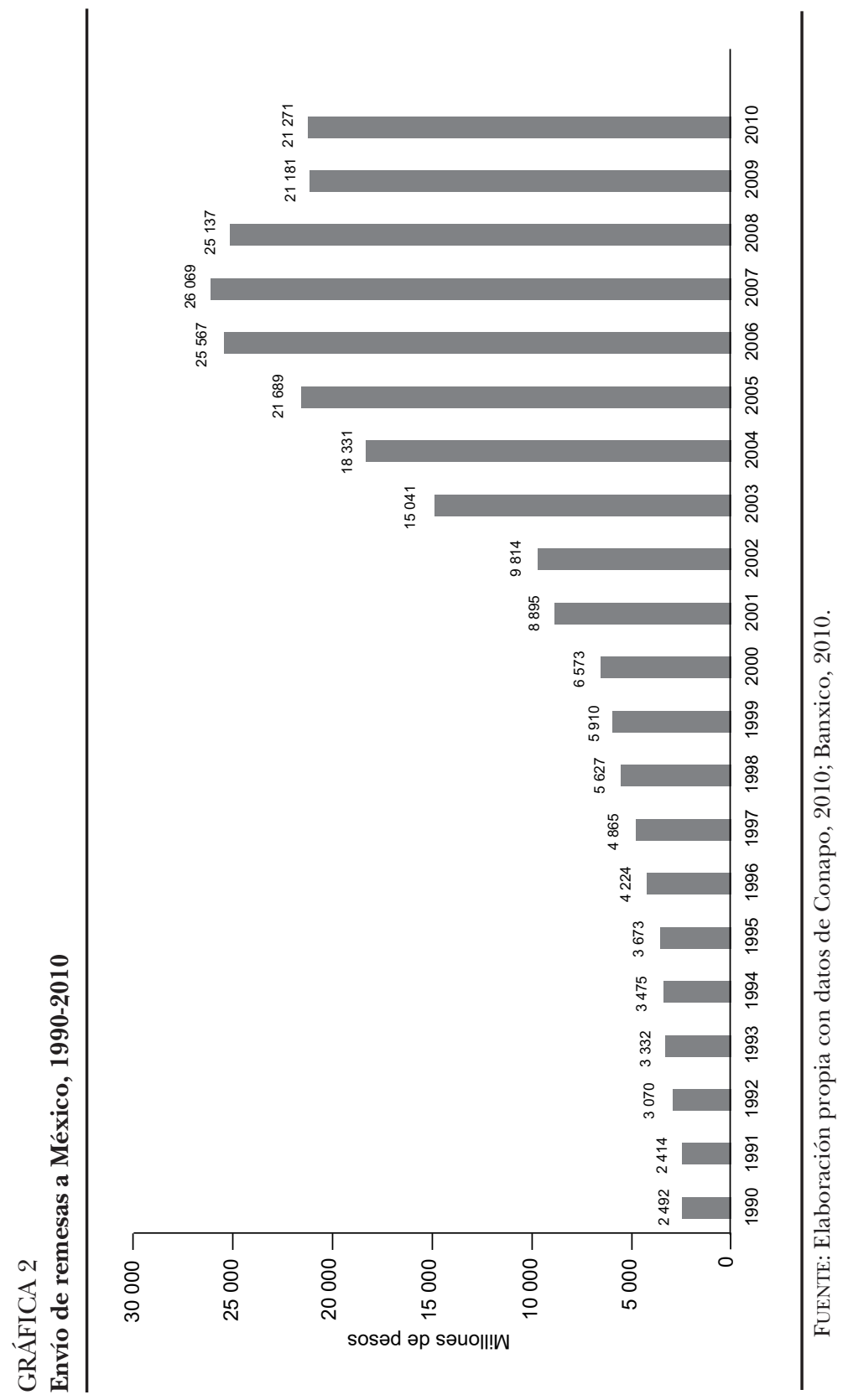


cuando se recibe un flujo de ingreso incierto; las de Hall (1978 y 1988) y Hey y Valentino (1988) acerca de la capacidad de consumo en el largo plazo en presencia de incertidumbre; las de Miller (1974), Hansen y Singleton (1983), Breeden (1986) y Bodie y Detemple (2004) sobre la maximización de la utilidad esperada de un inversionista cuando existe incertidumbre; y por último, las de Cox y Huang (1989) acerca de los flujos de ingresos no asegurables empleando programación dinámica.

En la siguiente sección se desarrolla un modelo estocástico en el que se plantea el problema del migrante que tiene un consumo de subsistencia sujeto a un ingreso incierto que sigue un movimiento geométrico browniano. El problema de decisión se resuelve por medio de la ecuación de Hamilton-Jacobi-Bellman. El modelo determina la proporción óptima de la riqueza que se reserva para el ahorro cuando éste es posible, las horas que destinará a su actividad laboral y la regla de consumo óptimo. En la sección 3 se revisa la evidencia empírica en las variables relevantes del modelo con datos sobre el migrante mexicano. En la última sección se presentan las conclusiones con observaciones acerca de las ventajas y limitaciones del modelo propuesto y se plantean futuros tópicos de investigación.

\section{El modelo estocástico del migrante}

El modelo propuesto amplía el trabajo de Constantinides (1990) al incorporar la posibilidad de que el individuo realice un ahorro, cuando hay un excedente, para suavizar su patrón de consumo (por la incertidumbre del mismo) y determinar cuántas horas está dispuesto a trabajar para incrementar su riqueza con el afán de maximizar su utilidad total esperada.

Se considera a un migrante como agente representativo de la población de mexicanos que migran a Estados Unidos, el cual tiene una riqueza, $W_{t}$, en el momento $t$ denominada en unidad de bienes de consumo; asimismo tiene acceso a un activo (ahorro), $H_{t}$, que paga una tasa de interés libre de riesgo en Estados Unidos, $r$, que se supone continuamente capitalizable y constante para todos los plazos; la dinámica del depósito es $H_{t}=H_{s} e^{r(t-s)}$ y satisface ${ }^{6}$

\footnotetext{
${ }^{6}$ Todo el análisis empírico para el migrante mexicano se realizará en pesos, por lo que la tasa de T-bills se transformará en una equivalente para México.
} 


$$
\frac{\mathrm{d} H_{t}}{H_{t}}=r \mathrm{~d} t
$$

si hay un excedente; en caso contrario $H_{t}=0$. Se supone que el salario, ${ }^{7}$ $\varphi_{t}$, es conducido por un movimiento geométrico browniano, es decir,

$$
\varphi_{t}=\varphi_{0} \exp \left[\left(\mu-\frac{1}{2} \sigma^{2}\right) t+\sigma V_{t}\right],
$$

donde $\mu$ es la tasa de crecimiento medio anualizado del salario incierto, $\sigma$ es la desviación estándar instantánea (volatilidad) de la tasa de crecimiento del salario y $V_{t}$ es un movimiento browniano (una variable aleatoria normal con media cero y varianza proporcional al tiempo). De esta manera, la ecuación diferencial estocástica que conduce al salario $\varphi_{t}$ está dada por:

$$
\frac{\mathrm{d} \varphi_{t}}{\varphi_{t}}=\mu \mathrm{d} t+\sigma \mathrm{d} V_{t}
$$

\section{Problema del migrante}

El problema de decisión que el migrante representativo desea resolver se plantea a continuación. La acumulación de la riqueza, $W_{t}$, en términos de los depósitos (ahorro), el ingreso y el consumo está dada por la siguiente ecuación diferencial:

$$
\left\{\begin{array}{l}
\mathrm{d} W_{t}=W_{t}\left(1-\alpha_{t}\right) \mathrm{d} R_{H}+W_{t} \alpha_{t} \mathrm{~d} R_{\varphi}-C_{t} \mathrm{~d} t-T_{t} \\
W_{t} \alpha_{t}=l_{t} \varphi_{t}
\end{array}\right.
$$

donde $C_{t}$ es el consumo, $T_{t}$ son las remesas (exógenamente determinadas) que envía el migrante a su familia, y $\mathrm{d} R_{\varphi}=\mathrm{d} \varphi_{t} / \varphi_{t}$ es la tasa de crecimiento del salario. De esta forma el migrante estaría dispuesto a trabajar $l_{t}$ horas para obtener un ingreso real, $l_{t} \varphi_{t}$, el cual representará la proporción de su riqueza, $W_{t} \alpha_{t}$, que proviene de su ingreso incierto. La proporción restante de su riqueza, $W_{t}\left(1-\alpha_{t}\right)$, la deposita en cajas de ahorro sólo cuando hay un excedente. La riqueza marginal del individuo, a su vez, es reducida por el consumo por unidad de tiempo,

\footnotetext{
7 Si bien el salario es fijo, depende de las horas que trabaje el migrante.
} 
$C_{t} \mathrm{~d} t$. El principal objetivo es determinar la proporción óptima de la riqueza que se destina al ahorro, las horas que el individuo está dispuesto a trabajar para incrementar su riqueza y su regla de consumo óptimo. Si se sustituyen las expresiones [1] y [2] en la ecuación diferencial [3] se tiene que:

$$
\mathrm{d} W_{t}=W_{t}\left[r+(\mu-r) \alpha_{t}-T_{t}-\frac{C_{t}}{W_{t}}\right] \mathrm{d} t-W_{t} \alpha_{t} \sigma \mathrm{d} V_{t}
$$

donde $0 \leq \alpha_{t} \leq 1$ y $W_{t} \geq 0$. El nivel de consumo de subsistencia está dado por:

$$
\Psi_{t} \equiv e^{-a t} \Psi_{0}+b \int_{0}^{t} e^{a(s-t)} C_{s} \mathrm{~d} s
$$

donde $C_{t}$ denota el consumo individual al tiempo $t, \Psi_{0}$ el consumo individual inicial de subsistencia, y $a, b$, son constantes; cuanto mayor sea el valor de $a$, menor será el peso del consumo pasado en la determinación de $\Psi_{t}$. El parámetro $b$ mide la fuerza de la formación de hábitos. Asimismo este agente sigue un proceso recursivo de formación de hábitos en su consumo $\Psi_{t}$, donde el consumo de subsistencia está relacionado con consumos anteriores (véase el apéndice A), de tal manera que:

$$
\mathrm{d} \Psi_{t}=b C_{t}-a \Psi_{t} .
$$

En el modelo propuesto las decisiones del migrante dependen de la información a tiempo $t$, el consumo debe ser mayor que el hábito $C_{t} \geq \Psi_{t}$, no puede haber un consumo cero y la riqueza debe ser mayor que cero, $W_{t} \geq 0$.

La función de utilidad del consumo esperada de un migrante racional se define como:

$$
\underset{C_{\mathrm{t}}}{\operatorname{Maximizar}} \quad \mathrm{E}_{0}\left\{\int_{0}^{\infty} u\left(C_{t}, \Psi_{t}\right) e^{-\rho s} \mathrm{~d} s \mid \mathrm{F}_{0}\right\} .
$$

En particular se toma

$$
u\left(C_{t}, \Psi_{t}\right)=\frac{\left(C_{t}-\Psi_{t}\right)^{\lambda}}{\lambda}
$$

como función de utilidad o satisfacción por el consumo y su rutina, donde $\lambda \neq 0$ está relacionado con la aversión al riesgo individual, $\Psi_{t}$ 
representa el nivel de hábito del bien, $\mathrm{F}_{t}$ es la información disponible al tiempo $t$, y $\rho$ es la tasa subjetiva de descuento; esta tasa es un indicador de qué tan ansioso está el migrante por su consumo presente. ${ }^{8}$ Un concepto relevante en este trabajo es el consumo de subsistencia, $C_{t}-\Psi_{t}$, el cual está relacionado con el consumo que se ha realizado anteriormente.

\section{La ecuación Hamilton-Jacobi-Bellman}

La solución óptima para el problema del migrante de la maximización de [6] sujeto a sus restricciones presupuestales [4] y [5] se obtiene con programación dinámica estocástica. Para ello se define la siguiente función valor:

$$
J\left(W_{t}, \Psi_{t}, t\right)=\max _{C_{s}, \alpha_{s}} \mathrm{E}\left[\int_{t}^{\infty} \frac{\left(C_{s}-\Psi_{s}\right)^{\lambda}}{\lambda} e^{-\rho s} \mathrm{~d} s \mid \mathrm{F}_{t}\right]
$$

Se separa el intervalo de integración de la anterior ecuación conduciendo a la relación de recursividad temporal sobre la función $J$ entre $(t, t+\mathrm{d} t)$ y $(t+\mathrm{d} t, \infty)$, de tal forma que

$$
J\left(W_{t}, \Psi_{t}, t\right)=\max _{c_{s}, \alpha_{s}} \mathrm{E}\left[\int_{t}^{t+\mathrm{d} t} \frac{\left(C_{s}-\Psi_{s}\right)^{\lambda}}{\lambda} e^{-\rho s} \mathrm{~d} s+\int_{t+\mathrm{d} t}^{\infty} \frac{\left(C_{s}-\Psi_{s}\right)^{\lambda}}{\lambda} e^{-\rho s} \mathrm{~d} s \mid \mathrm{F}_{t}\right]
$$

Se aplica al primer término el teorema de valor medio del cálculo integral y al segundo la definición de diferencial, de tal manera que

$$
J\left(W_{t}, \Psi_{t}, t\right)=\max _{C_{t}, \alpha_{t}} \mathrm{E}\left[\frac{\left(C_{t}-\Psi_{t}\right)^{\lambda}}{\lambda} e^{-\rho t} \mathrm{~d} t+\mathrm{o}(\mathrm{d} t)+J\left(W_{t}, \Psi_{t}, t\right)+\mathrm{d} J\left(W_{t}, \Psi_{t}, t\right) \mid \mathrm{F}_{t}\right][9]
$$

Posteriormente se aplica el lema de Itô a $J_{t}$ y se considera el sistema de ecuaciones diferenciales estocásticas dadas por:

$$
\left\{\begin{array}{l}
\mathrm{d} W_{t}=W_{t}\left[r+(\mu-r) \alpha_{t}-T_{t}-\frac{C_{t}}{W_{t}}\right] \mathrm{d} t-W_{t} \alpha_{t} \sigma \mathrm{d} V_{t} \\
\mathrm{~d} \Psi_{t}=\left(b C_{t}-a \Psi_{t}\right)+0
\end{array}\right.
$$

${ }^{8}$ En otras palabras, entre mayor sea $\rho$, más ansioso estará el agente por consumir en el presente que en el futuro. 
El lema de Itô (véase el apéndice B) conduce a:

$$
\begin{aligned}
\mathrm{d} J= & {\left[\frac{\partial J}{\partial t}+\frac{\partial J}{\partial W_{t}}\left(r+(\mu-r) \alpha_{t}-T_{t}-\frac{C_{t}}{W_{t}}\right)+\frac{1}{2} \frac{\partial^{2} J}{\partial W_{t}^{2}} W_{t}^{2} \alpha_{t}^{2} \sigma^{2}\right.} \\
& \left.+\frac{\partial J}{\partial \Psi_{t}}\left(b C_{t}-a \Psi_{t}\right)\right] \mathrm{d} t+\frac{\partial J}{\partial W_{t}} W_{t} \alpha_{t} \sigma \mathrm{d} V_{t}
\end{aligned}
$$

De esta manera [9] se transforma en:

$$
\begin{array}{rl}
0=\max _{c_{t}, \alpha_{t}} & \mathrm{E}\left\{\frac{\left(C_{t}-\Psi_{t}\right)^{\lambda}}{\lambda} e^{-\rho t} \mathrm{~d} t+\mathrm{o}(\mathrm{d} t)+\left[\frac{\partial J}{\partial t}+\frac{\partial J}{\partial W_{t}}\left(r+(\mu-r) \alpha_{t}-T_{t}-\frac{C_{t}}{W_{t}}\right)\right.\right. \\
+ & \left.\left.\frac{1}{2} \frac{\partial^{2} J}{\partial W_{t}^{2}} W_{t}^{2} \sigma^{2} \alpha_{t}^{2}+\frac{\partial J}{\partial \Psi_{t}}\left(b C_{t}-a \Psi_{t}\right)\right] \mathrm{d} t+\frac{\partial J}{\partial W_{t}} W_{t} \alpha_{t} \sigma \mathrm{d} V_{t} \mid \mathrm{F}_{t}\right\}
\end{array}
$$

A continuación se toman los valores esperados en todos los términos de la ecuación, se divide entre $\mathrm{d} t$ y se toma el límite $\mathrm{de} o(\mathrm{~d} t) / \mathrm{d} t \rightarrow 0$, así

$$
\begin{aligned}
0=\max _{c_{t}, \alpha_{t}} & {\left[\frac{\left(C_{t}-\Psi_{t}\right)^{\lambda}}{\lambda} e^{-\rho t}+\frac{\partial J}{\partial t}+\frac{\partial J}{\partial W_{t}}\left(r+(\mu-r) \alpha_{t}-T_{t}-\frac{C_{t}}{W_{t}}\right)\right.} \\
& \left.+\frac{1}{2} \frac{\partial^{2} J}{\partial W_{t}^{2}} W_{t}^{2} \sigma^{2} \alpha_{t}^{2}+\frac{\partial J}{\partial \Psi_{t}}\left(b C_{t}-a \Psi_{t}\right)\right]
\end{aligned}
$$

Si $C_{t}$ y $\alpha_{t}$ son óptimos se obtiene la ecuación diferencial parcial de segundo orden en $J$.

$$
\begin{aligned}
0= & \frac{\left(C_{t}-\Psi_{t}\right)^{\lambda}}{\lambda} e^{-\rho t}+\frac{\partial J}{\partial t}+\frac{\partial J}{\partial W_{t}}\left(r+(\mu-r) \alpha_{t}-T_{t}-\frac{C_{t}}{W_{t}}\right)+\frac{1}{2} \frac{\partial^{2} J}{\partial W_{t}^{2}} W_{t}^{2} \sigma^{2} \alpha_{t}^{2} \\
& +\frac{\partial J}{\partial \Psi_{t}}\left(b C_{t}-a \Psi_{t}\right)
\end{aligned}
$$

Se propone como candidato de solución de la ecuación diferencial parcial anterior a

$$
J\left(W_{t}, \Psi_{t}, t\right)=\mathrm{V}\left(W_{t}, \Psi_{t}\right) e^{-\rho t}=\beta_{1} \frac{\left(W_{t}-\beta_{0} \Psi_{t}\right)^{\lambda}}{\lambda} .
$$


Para este candidato se calculan las derivadas parciales, de esta manera se transforma en:

$$
\begin{gathered}
0=\frac{\left(C_{t}-\Psi_{t}\right)^{\lambda}}{\lambda}-\rho \beta_{1} \frac{\left(W_{t}-\beta_{0} \Psi_{t}\right)^{\lambda}}{\lambda}+\beta_{1}\left(W_{t}-\beta_{0} \Psi_{t}\right)^{\lambda-1} W_{t}\left(r+(\mu-r) \alpha_{t}-T_{t}-\frac{C_{t}}{W_{t}}\right) \\
+\frac{1}{2} \beta_{1}(\lambda-1)\left(W_{t}-\beta_{0} \Psi_{t}\right)^{\lambda-2} W_{t}^{2} \sigma^{2} \alpha_{t}^{2}-\beta_{0} \beta_{1}\left(W_{t}-\beta_{0} \Psi_{t}\right)^{\lambda-1}\left(b C_{t}-a \Psi_{t}\right)
\end{gathered}
$$

Si se deriva con respecto a las variables de control $C_{t}$ y $\alpha_{t}$ de la ecuación anterior se tiene que:

$$
C_{t}^{*}=\beta_{1}^{\frac{1}{\lambda-1}}\left(W_{t}-\beta_{0} \Psi_{t}\right)\left(1+b \beta_{0}\right)^{\frac{1}{\lambda-1}}+\Psi_{t},
$$

y

$$
\alpha_{t}^{*}=\frac{(\mu-r)}{\sigma_{t}^{2}(1-\lambda)} \frac{1}{W_{t}}\left(W_{t}-\beta_{0} \Psi_{t}\right),
$$

donde $0<\lambda<1$.

Ahora bien, si se sustituyen los valores de $\beta_{0}$ y $\beta_{1}$ (véase el apéndice C) en las ecuaciones [14] y [15], respectivamente, se tiene que:

$$
C_{t}^{*}=\Psi_{t}+h\left(W_{t}-\frac{\Psi_{t}}{r+a-b}\right),
$$

donde $h$ es una constante. Este resultado muestra la trayectoria de consumo óptimo que el migrante seguirá en el futuro, ya que satisface sus necesidades básicas de sobrevivencia. Es decir, el consumo del individuo tiene que ser por lo menos $\Psi_{t}$ y si la riqueza $W_{t}$ excede un umbral que depende de los parámetros que determinan la formación de hábitos. Es importante mencionar que la cantidad

$$
h=\left(\beta_{1}\left(1+b \beta_{0}\right)\right)^{\frac{1}{\lambda-1}}=\left[\left(\frac{1}{(1-\lambda)(r+a-b)-\lambda}\right)^{\lambda-1}\left(1-\frac{b}{r+a-b}\right)\right]^{\frac{1}{\lambda-1}}
$$

es constante en el tiempo y representa la propensión marginal al consumo después del consumo de subsistencia. En lo que sigue se supondrá que la tasa de interés es mayor, $a-b$. La cantidad 


$$
\alpha_{t}^{*}=m\left(1-\frac{\frac{\Psi_{t}}{W_{t}}}{r+a-b}\right),
$$

representa la proporción óptima del salario incierto que el migrante está dispuesto a incluir en su riqueza y que asegura que siga haciendo depósitos en cajas de ahorro. La constante $m$ está dada por

$$
m=\frac{(\mu-r)}{(1-\lambda)} \frac{1}{\sigma^{2}} \text {. }
$$

A continuación se obtiene el proceso estocástico que genera la riqueza real del consumidor cuando las decisiones óptimas son aplicadas. Después de sustituir la proporción óptima en la ecuación [3], se obtiene:

$$
\mathrm{d} W_{t}=W_{t}\left[r+(\mu-r) \alpha_{t}^{*}-T_{t}-\frac{C_{t}^{*}}{W_{t}}\right] \mathrm{d} t-W_{t} \alpha_{t}^{*} \sigma \mathrm{d} V_{t}
$$

y

$$
l_{t}=\frac{W_{t} \alpha_{t}^{*}}{\varphi_{t}}
$$

Si se retoma que $\varphi_{t}=\varphi_{0} e^{\left(\mu-\frac{1}{2} \sigma^{2}\right) t+\sigma V_{t}}$

$$
\begin{gathered}
l_{t}=\varphi_{0} e^{-\left(\mu-\frac{1}{2} \sigma^{2}\right) t-\sigma V_{t}}\left(1-\frac{\frac{\Psi_{t}}{W_{t}}}{r+a-b}\right) \\
\left(W_{0}+\int_{0}^{t} W_{s}\left[r+(\mu-r) \alpha_{s}^{*}-T_{t}-\frac{C_{s}^{*}}{W_{s}}\right] \mathrm{d} s-\int_{0}^{t} W_{s} \alpha_{s}^{*} \sigma \mathrm{d} V_{s}\right)
\end{gathered}
$$

$l_{t}$ representa las horas que el migrante debe trabajar para ganar un salario que le permita destinar una parte a su riqueza y mantener su consumo de subsistencia.

Al sustituir [20] en la ecuación [19] se obtiene que la riqueza depende de las horas que trabaja el migrante a cambio de un salario, y si hay un sobrante lo ahorra en cajas de ahorro. 


$$
\mathrm{d} W_{t}=W_{t}\left[r+(\mu-r) \alpha_{t}^{*}-T_{t}-\frac{C_{t}^{*}}{W_{t}}\right] \mathrm{d} t-l_{t} \sigma \mathrm{d} V_{t}
$$

En este caso se supone que $T_{t}=T_{0} e^{\zeta t}$, donde $\zeta$ es la tasa de crecimiento de las reservas, la cual puede ser positiva, negativa, e incluso dependiente del tiempo $\zeta=\zeta(t)$, que es exógenamente determinada al realizar el primer algoritmo Lozano (1998).

\section{Evidencia empírica sobre el modelo}

A continuación se presenta evidencia empírica para fortalecer el modelo propuesto presentado anteriormente para un migrante mexicano que vive en Estados Unidos. Para ello se consideran ciertas variables como el consumo (C), el salario del migrante tras la conversión de dólares a pesos al tipo de cambio de 2012 (S), la tasa interés que se paga en México por hacer depósitos de ahorro (I) y la variable más importante, que son las remesas por migrante enviadas a México desde Estados Unidos (RM).

El presente análisis utiliza series de tiempo para el periodo de 1980 a 2011 de forma trimestral. Los datos faltantes fueron estimados por el método de simulación Monte Carlo, ya que en ocasiones no existen datos sobre las variables a analizar.

Se aplican modelos de vectores autorregresivos (VAR), que son una generalización de los modelos AR: en general una variable se explica en función de sus valores pasados, y su amplio uso se debe a la simplificación de varios supuestos que requieren los modelos de orden estructural. No es necesario establecer la condición de endogeneidadexogeneidad, pues se definen todas las variables como endógenas. Para tal efecto el marco metodológico consiste en seis pruebas econométricas: $a$ ) análisis de raíces unitarias, $b$ ) análisis de cointegración, c) modelo de causalidad de Granger, $d$ ) vector autorregresivo, $e$ ) funciones de impulso-respuesta, y f) análisis de descomposición de la varianza, en el que se analiza cómo interactúan principalmente las variables RP y $\mathrm{S}$ ante el consumo del migrante. 


\section{Análisis de raíces unitarias}

Para analizar las relaciones entre dos o más variables es necesario precisar si los procesos estocásticos que generan las series de tiempo son invariables en el tiempo, esto es, si dichos procesos son estacionarios, a fin de no concluir erróneamente proponiendo relaciones espurias entre las variables bajo análisis. Se requiere para ello que las series carezcan de raíces unitarias y que sean por lo tanto del orden $I(0)$.

La prueba más comúnmente aplicada es la Dickey-Fuller Aumentada (ADF), pero para fortalecer el estudio se utilizaron las demás pruebas disponibles: Dickey-Fuller GIS (ERS) y Phillips-Perron (PP), que se muestran en el cuadro 1, en el cual se observa que los resultados de las pruebas mencionadas anteriormente para las series de C, S, I, RM en primeras diferencias son estacionarias.

En todos los casos el rechazo a la hipótesis nula es fuerte. El estadístico $t$ obtenido supera todos los valores críticos y señala el rechazo de la existencia de raíz unitaria aun en el exigente nivel de confianza de $1 \%$. Además, la probabilidad de MacKinnon en todos los casos es menor a 0.05 por ciento.

\section{Análisis de cointegración}

Al aplicar el modelo de Johansen los resultados son favorables; por un lado el estadístico de la traza es mayor que los valores críticos a un nivel de $5 \%$ de significancia, por lo que se rechaza la hipótesis nula de la existencia de vectores cointegrantes, y por otro lado en la prueba del máximo eingenvalor las conclusiones son similares a las de la prueba de la traza y se confirma, según el máximo eingenvalor, que hay más de un vector de cointegración. De acuerdo con la prueba de Johansen, C, S, I, RM sostienen una relación de equilibrio de largo plazo entre ellas.

\section{Análisis de causalidad Granger}

La prueba de causalidad de Granger busca determinar estadísticamente si el pasado de una variable contiene información que preceda al comportamiento de otra variable y que, por lo tanto, contribuye a explicarla y viceversa. 


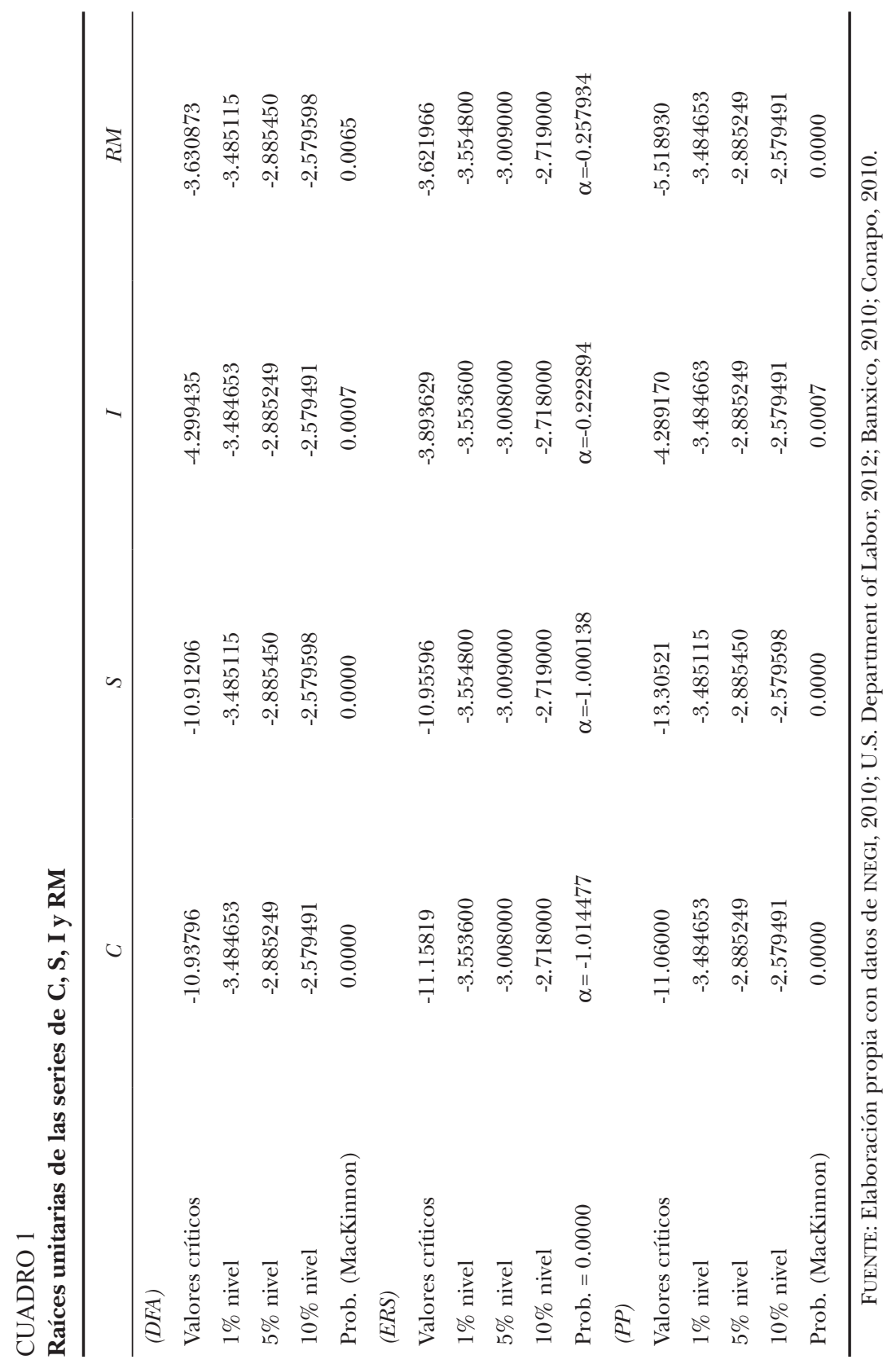




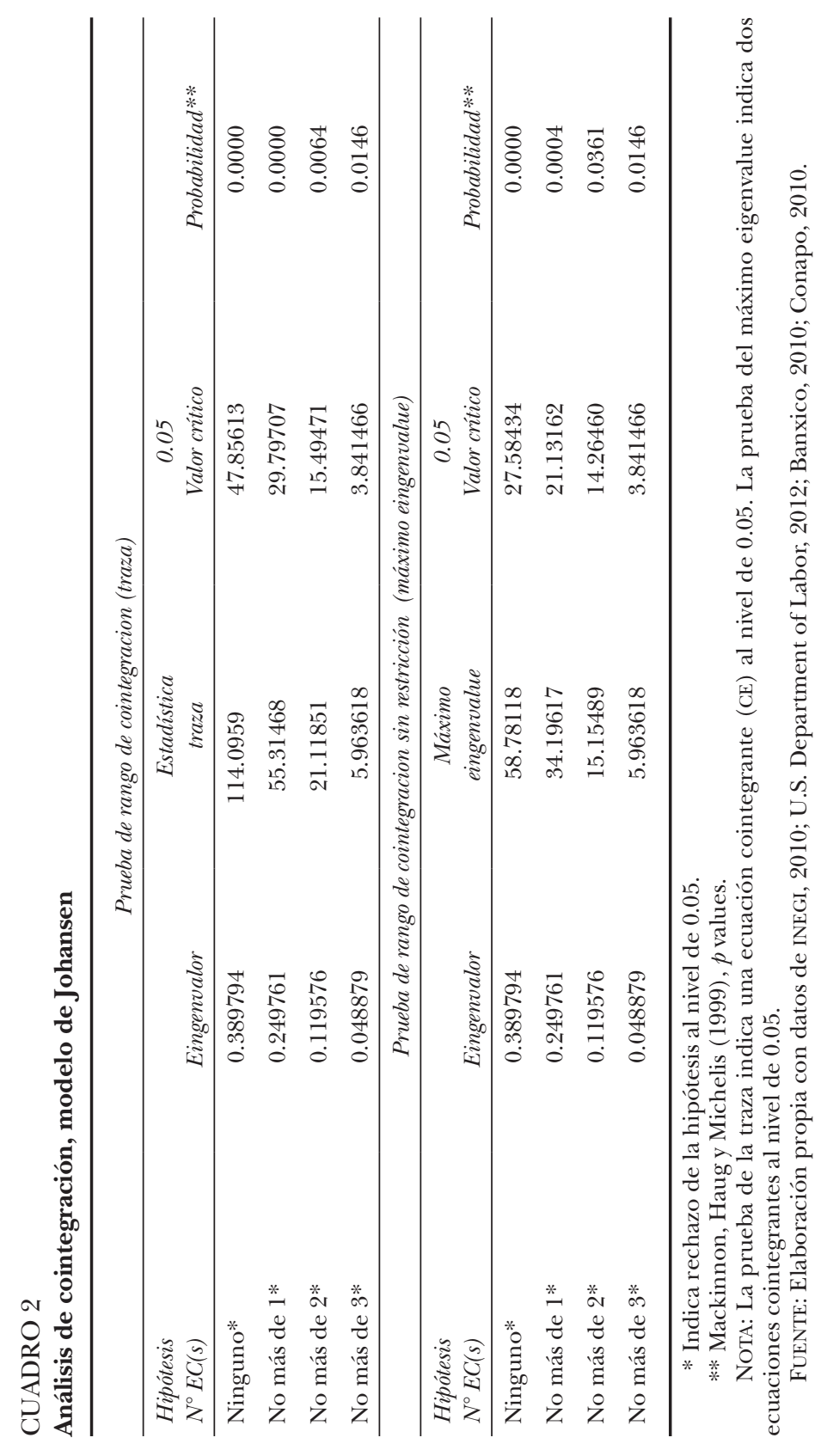


Cabe aclarar que la causalidad de Granger es muy sensible al número de rezagos si el valor estadístico $F$ supera el valor tabulado; se rechaza la hipótesis nula y por tanto se acepta que una variable causa a otra o viceversa. La causalidad de Granger se presenta en el cuadro 3 , donde se identifica que es bidireccional entre las variables. Esto significa que C, S, I, RM influyen entre las variables antes mencionadas.

\section{Vector autorregresivo}

La metodología VAR está estrechamente ligada con la cointegración que se analizó en la sección anterior, la cual indica que si existe cointegración entonces hay necesariamente una representación de corto plazo que corrige el error y evita que las series cointegradas se dispersen en el tiempo. Pero también se plantea que la cointegración es una condición necesaria para que se pueda realizar un modelo de corrección de error. De esta forma se combina la idea de encontrar una relación estable de largo plazo con los ajustes estadísticos de los desequilibrios de corto plazo.

Para estimarlo es preciso identificar el grado de integración de las variables $\mathrm{I}(1)$, el cual, presentando el mismo orden de integración, cuenta con información histórica relevante. Un factor importante del análisis en el VAR es el número de rezagos a emplearse, por lo que es necesario evaluar uno a uno cada rezago hasta encontrar el número óptimo contrastando los criterios de información de los resultados que genera. En este trabajo se utiliza el criterio de Akaike y Schwarz, que en general muestra resultados muy parecidos o incluso idénticos. ${ }^{9}$ En este caso se toma el criterio que ofrezca menos rezagos debido a que un incremento de rezagos disminuye $R^{2}$.

Otra característica importante es la estabilidad del VAR, que se puede observar mediante el valor de sus raíces características, en el que los valores son menores que 1, y en la representación de la gráfica 3 se encuentran dentro del círculo unitario. Con esto se asegura que ante choques de corto plazo las variables regresen a su trayectoria de equilibrio de largo plazo. Si no fuera éste el caso se tendría un modelo explosivo, lo que carece de sentido económico. Para verificar la estabilidad se debe examinar si las raíces son menores que 1 en valor absoluto. En el cuadro 4 se puede observar el rezago óptimo para el VAR, que es 1.

9 Ocasionalmente los resultados pueden ser muy distintos. 


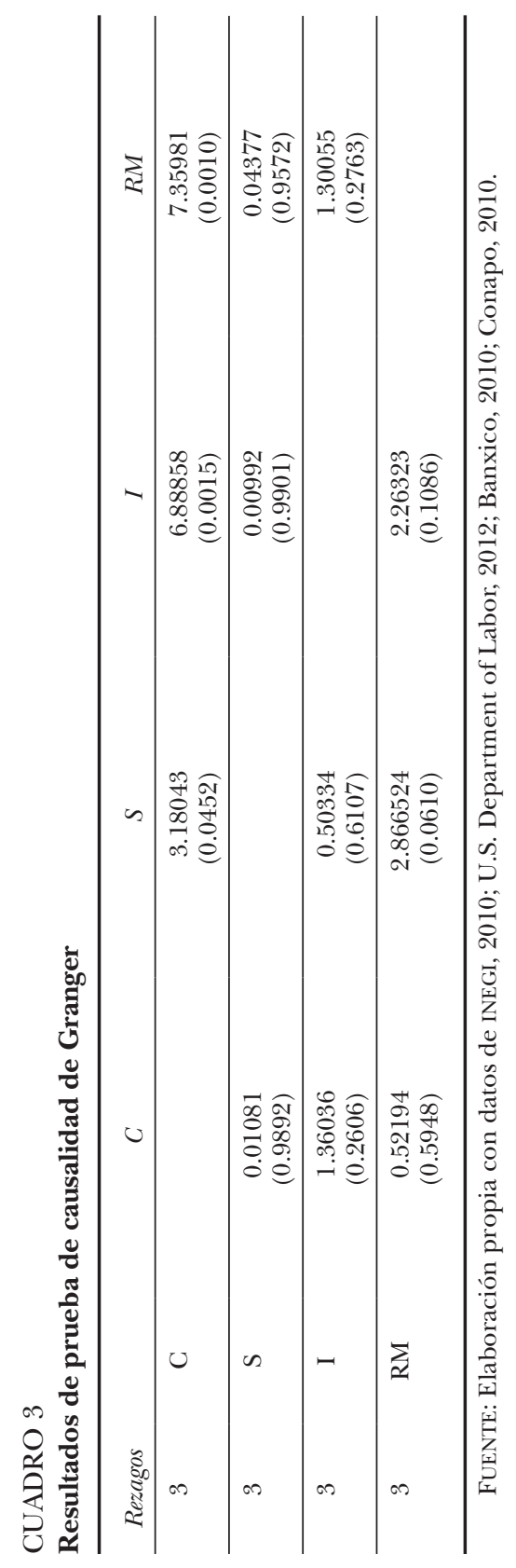




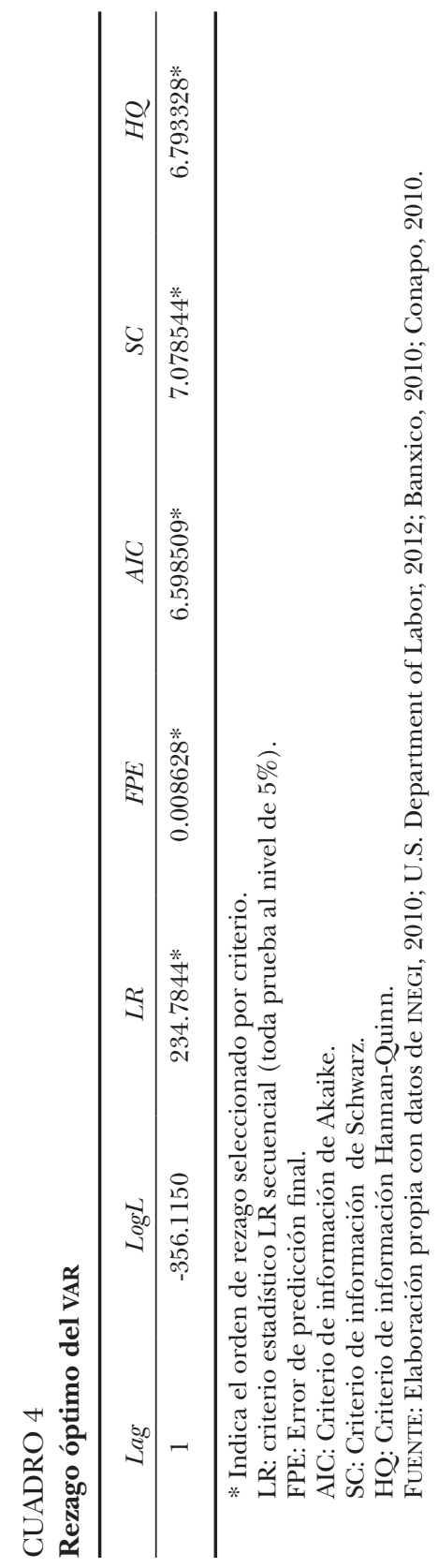




\section{GRÁFICA 3}

Círculo de raíces unitarias (raíces inversas del polinomio característico AR)

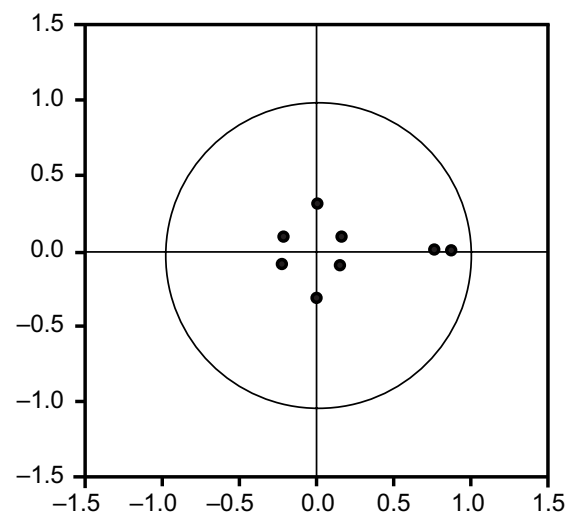

FUENTE: Elaboración propia con datos de INEGI, 2010; U.S. Department of Labor, 2010; Banxico, 2010; Conapo, 2010.

Una vez definido el VAR es preciso constatar que no se rechacen las pruebas de normalidad, heteroscedasticidad y estabilidad de los residuales. Tanto la normalidad como la heteroscedasticidad pasan las mismas condiciones de estimación de MCO, es decir, su probabilidad es mayor que 0.05 , y se rechaza la hipótesis nula de no normalidad con 0.059 y la de no heteroscedasticidad con 0.5321 .

\section{Análisis de impulso-respuesta}

El análisis impulso-respuesta es útil para estudiar la congruencia y la sensibilidad dinámica de las variables especificadas en un modelo, atendiendo a que no haya covarianzas distintas de 0 , es decir, los errores tienen que ser no correlacionados, ya que en caso contrario sería imposible precisar la respuesta de una variable ante impulsos de otras variables específicas. El análisis indica la respuesta dinámica de la variable dependiente en el VAR ante cambios en los términos de error o innovaciones de todas las variables endógenas, excluyendo los efectos de las variables que expresamente se asignan como exógenas. 
Cabe resaltar que la respuesta solamente puede ser calculada si el VAR se encuentra en equilibrio de largo plazo. Por otro lado, se debe tomar en cuenta la longitud del cambio, ya que si éste se considera muy corto no se pueden observar con precisión la evolución de los cambios y la estabilidad dinámica del VAR. De esta manera, si el VAR es estable una perturbación hará que el sistema salga de su trayectoria de equilibrio, aunque después de algunos periodos regrese al equilibrio.

El análisis de la presente investigación se basa en un intervalo de 10 periodos a fin de observar el proceso de cambios y de ajustes de manera completa. Aunque el análisis impulso-respuesta requiere cuatro estudios (todas las variables contra todas), únicamente se examina el impulso de las cuatro variables sobre $\mathrm{C}$ para apreciar los efectos de las demás sobre ésta.

En la gráfica 4A se observa que dentro de este trabajo uno de los análisis más importantes es el impulso-respuesta de C ante RM y todos los periodos son significativos. Los primeros cuatro meses hay una respuesta negativa ante RM y después la respuesta es positiva, pero vuelve a ser negativa en los meses sexto y séptimo, aunque posteriormente llega a un punto de equilibrio.

La gráfica 4B muestra el otro análisis de interés: el de C ante impulsos de $\mathrm{S}$, el cual comienza con un impacto negativo del primero al quinto periodos, se vuelve positivo en el sexto mes y negativo en el octavo; posteriormente comienza a desaparecer el impulso y zigzaguea hasta llegar a la estabilidad.

En la gráfica 4C se presenta la respuesta de C ante impulsos de I: comienza con un impacto negativo del primero al tercer periodo, se vuelve positivo en el cuarto mes y negativo del sexto al octavo; posteriormente comienza a estabilizarse.

\section{Análisis de descomposición de la varianza}

El estudio de la descomposición de la varianza es complementario al análisis impulso-respuesta que informa en distintos horizontes del tiempo el porcentaje de volatilidad que registra una variable por los choques de las demás. De esta manera es posible medir la volatilidad que genera la variable endógena en la variable exógena en un momento específico. En el cuadro 5 se resumen los resultados para el primero y decimosegundo periodos. Se observa que todas las variables I, RM tienen un fuerte comportamiento autorregresivo, esto en virtud de 


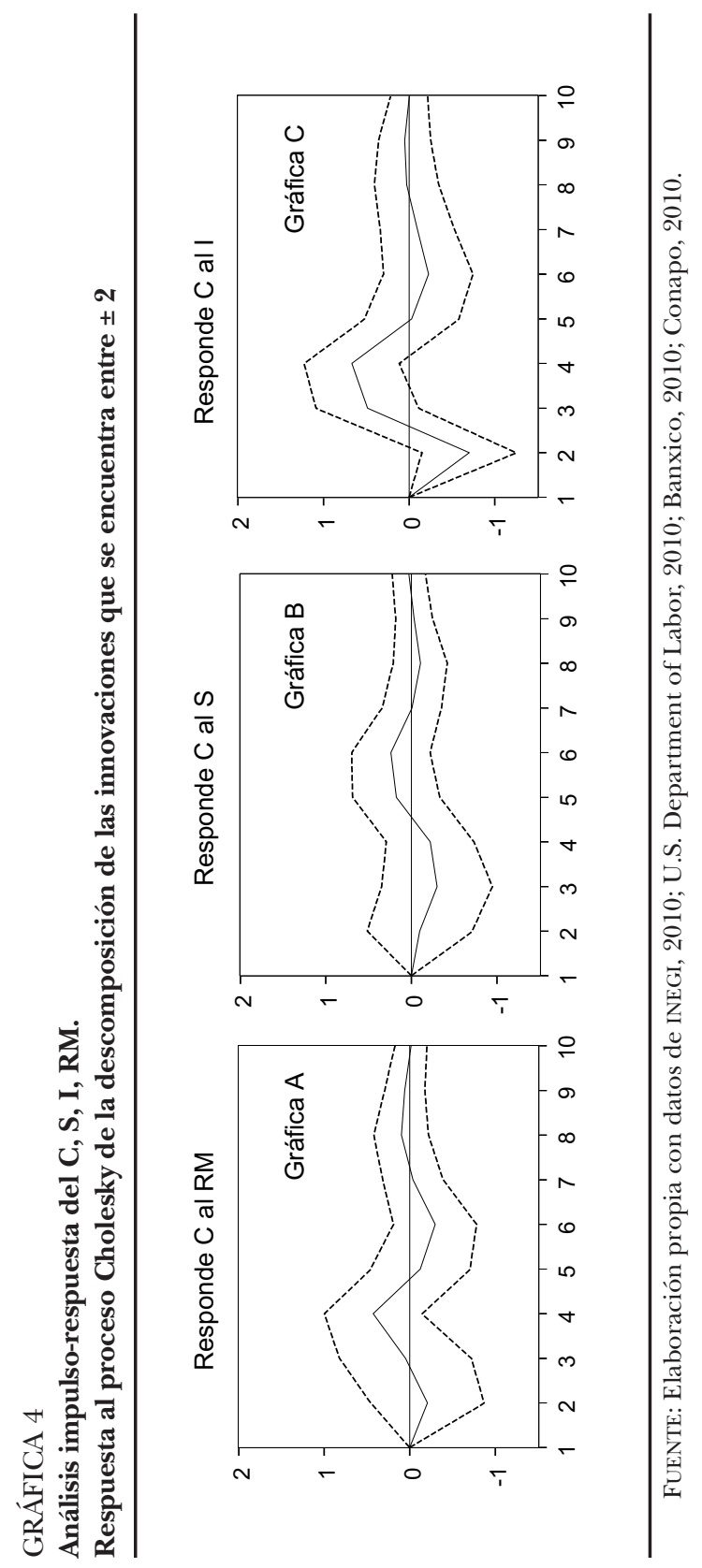




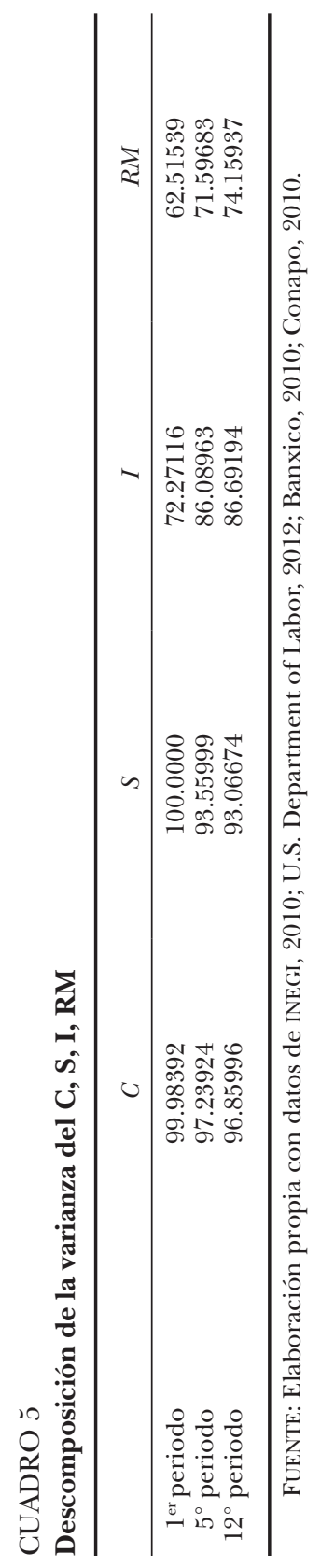


que después de 12 meses más de $74 \%$ de la varianza de cada variable se sigue explicando por sí mismo.

Los diferentes análisis empíricos evidencian que sí se mantiene una relación de equilibrio de largo plazo. El análisis de causalidad de Granger confirma una relación bidireccional entre las variables. El análisis del vector autorregresivo confirma la cointegración a corto plazo, en tanto las funciones de impulso-respuesta indican impulsos positivos y negativos, y en todos los casos zigzaguean y se estabilizan en alrededor del decimoquinto mes.

\section{Conclusiones}

En este trabajo se desarrolló un modelo estocástico sobre el comportamiento de un migrante mexicano que vive en Estados Unidos (de hecho puede tratarse de cualquier agente, sea migrante o no) y que desea mantener su consumo de subsistencia sujeto a las horas que trabaja ganando un ingreso incierto. La formación de hábitos es parte importante de la toma de decisiones de este agente racional, pues su consumo pasado sí influye en su consumo futuro (su hábito) y de subsistencia.

La estrategia de consumo óptima acarrea cambios importantes en las decisiones del migrante sobre su consumo y se convierte en una variable aleatoria, situación que es más acorde con la realidad. La formación de hábitos se debe incorporar en los próximos modelos tanto financieros como económicos para observar cómo reaccionan los individuos en su consumo futuro tomando en cuenta el consumo pasado.

Asimismo, para fortalecer los hallazgos teóricos que se encontraron para el migrante mexicano se realizó un análisis econométrico con datos del salario y remesas del migrante representativo y de otras variables relevantes. Los diferentes análisis econométricos evidencian que sí se mantiene una relación de equilibrio de largo plazo. El análisis de causalidad de Granger fortalece una relación bidireccional entre las variables. El análisis del vector autorregresivo confirma la cointegración a corto plazo, en tanto las funciones de impulso-respuesta indican impulsos positivos y negativos significativos de las variables salario y remesas del migrante ante el consumo en todos los casos, y zigzaguean para estabilizarse alrededor del decimoquinto mes. Por último, la descomposición de la varianza demuestra que la volatilidad 
de las variables se sigue explicando a sí misma al presentarse desde el primero hasta el último mes del periodo analizado.

\section{APÉNDICE A}

\section{Dinámica de formación de hábitos}

La dinámica de formación de hábitos se puede expresar como:

$$
\begin{gathered}
\Psi_{t}=b C_{t}+(1-a) \Psi_{t-1} \\
\Psi_{t}-\Psi_{t-\Delta}=b C_{t} \Delta-a \Psi_{t} \Delta \\
\frac{\Psi_{t}-\Psi_{t-\Delta}}{\Delta}=b C_{t}-a \Psi_{t} \\
\frac{\mathrm{d} \Psi}{\mathrm{d} t}=b C_{t}-a \Psi_{t}
\end{gathered}
$$

donde:

$C_{i}$ : consumo hoy

$\Psi_{t}$ : formación del hábito hoy

$\Psi_{t-1}$ : formación del hábito asociado al consumo pasado.

\section{APÉNDICE B}

\section{Lema de Itô para dos variables}

Considere un sistema de ecuaciones diferenciales estocásticas dadas por:

$$
\left\{\begin{array}{l}
\mathrm{d} S_{1 t}=\mu_{1}\left(S_{1 t}, t\right) \mathrm{d} t+\sigma_{1}\left(S_{1 t}, t\right) \mathrm{d} W_{1 t} \\
\mathrm{~d} S_{2 t}=\mu_{2}\left(S_{2 t}, t\right) \mathrm{d} t+\sigma_{2}\left(S_{2 t}, t\right) \mathrm{d} W_{2 t}
\end{array}\right.
$$

donde:

$$
\operatorname{Cov}\left(\mathrm{d} W_{1 t}, \mathrm{~d} W_{2 t}\right)=\rho \mathrm{d} t .
$$

Si se define una función $y=f\left(S_{1 t}, S_{2 t}, t\right)$, entonces la expansión de serie de Taylor de esta función hasta llegar en términos de segundo orden está dada por: 


$$
\begin{aligned}
\mathrm{d} y= & \frac{\partial f}{\partial t} \mathrm{~d} t+\frac{\partial f}{\partial S_{1 t}} \mathrm{~d} S_{1 t}+\frac{\partial f}{\partial S_{2 t}} \mathrm{~d} S_{2 t}+\frac{1}{2}\left[\frac{\partial^{2} f}{\partial t^{2}}(\mathrm{~d} t)^{2}+\frac{\partial^{2} f}{\partial S_{1 t}^{2}}\left(\mathrm{~d} S_{1 t}\right)^{2}\right. \\
& \left.+\frac{\partial^{2} f}{\partial S_{2 t}^{2}}\left(\mathrm{~d} S_{2 t}\right)^{2}+2\left(\frac{\partial^{2} f}{\partial S_{1 t} \partial t} \mathrm{~d} S_{1 t} \mathrm{~d} t+\frac{\partial^{2} f}{\partial S_{2 t} \partial t} \mathrm{~d} S_{2 t} \mathrm{~d} t+\frac{\partial^{2} f}{\partial S_{1 t} \partial S_{2 t}} \mathrm{~d} S_{1 t} \mathrm{~d} S_{2 t}\right)\right]
\end{aligned}
$$

Al sustituir la ecuación [B1] y la aplicación de las reglas básicas de diferenciación estocástica $(\mathrm{d} t)^{2}=0,(\mathrm{~d} t)\left(\mathrm{d} W_{t}\right)=0 \mathrm{y}\left(\mathrm{d} W_{t}\right)_{2}=\mathrm{d} t$, se obtiene lo que se conoce como el lema de Itô. En este caso para una función de dos variables o dos activos cada uno con su media y su varianza se tiene que:

$$
\begin{aligned}
\mathrm{d} y= & {\left[\frac{\partial f}{\partial t}+\frac{\partial f}{\partial S_{1 t}} \mu_{1}\left(S_{1 t}, t\right)+\frac{\partial f}{\partial S_{2 t}} \mu_{2}\left(S_{2 t}, t\right)+\frac{1}{2} \frac{\partial^{2} f}{\partial S_{1 t}^{2}} \sigma_{1}^{2}\left(S_{1 t}, t\right)\right.} \\
& \left.+\frac{1}{2} \frac{\partial^{2} f}{\partial S_{2 t}^{2}} \sigma_{2}^{2}\left(S_{2 t}, t\right)+\frac{\partial f}{\partial S_{1 t} \partial S_{2 t}} \rho \sigma_{1}\left(S_{1 t}, t\right) \sigma_{2}\left(S_{2 t}, t\right)\right] \mathrm{d} t \\
& +\frac{\partial f}{\partial S_{1 t}} \sigma_{1}\left(S_{1 t}, t\right) \mathrm{d} W_{1 t}+\frac{\partial f}{\partial S_{2 t}} \sigma_{2}\left(S_{2 t}, t\right) \mathrm{d} W_{2 t}
\end{aligned}
$$

\section{APÉNDICE $\mathrm{C}$}

\section{Cálculo de $\beta_{0}$ y $\beta_{1}$}

Si se sustituyen las ecuaciones [15] y [16] en [14] se obtienen los valores de $\beta_{0}$ y $\beta_{1}$.

$$
\begin{gathered}
0=\frac{\left(\left[\beta_{1}^{\frac{1}{\lambda-1}}\left(W_{t}-\beta_{0} \Psi_{t}\right)\left(1+b \beta_{0}\right)^{\frac{1}{\lambda-1}}+\Psi_{t}\right]-\Psi_{t}\right)^{\lambda}}{\lambda}-\rho \beta_{1} \frac{\left(W_{t}-\beta_{0} \Psi_{t}\right)^{\lambda}}{\lambda} \\
+\beta_{1}\left(W_{t}-\beta_{0} \Psi_{t}\right)^{\lambda-1} W_{t}\left(r+(\mu-r)\left[\frac{(\mu-r)}{\sigma_{t}^{2}(1-\lambda)} \frac{1}{W_{t}}\left(W_{t}-\beta_{0} \Psi_{t}\right)\right]\right. \\
\left.+T_{t}-\frac{\beta_{1}^{\frac{1}{\lambda-1}}\left(W_{t}-\beta_{0} \Psi_{t}\right)\left(1+b \beta_{0}\right)^{\frac{1}{\lambda-1}}+\Psi_{t}}{W_{t}}\right]+\frac{1}{2} \beta_{1}(\lambda-1)\left(W_{t}-\beta_{0} \Psi_{t}\right)^{\lambda-2} W_{t}^{2} \sigma^{2}
\end{gathered}
$$




$$
\begin{gathered}
{\left[\frac{(\mu-r)}{\sigma_{t}^{2}(1-\lambda)} \frac{1}{W_{t}}\left(W_{t}-\beta_{0} \Psi_{t}\right)\right]^{2}-\beta_{0} \beta_{1}\left(W_{t}-\beta_{0} \Psi_{t}\right)^{\lambda-1}} \\
\left(b\left[\beta_{1}^{\overline{\lambda-1}}\left(W_{t}-\beta_{0} \Psi_{t}\right)\left(1+b \beta_{0}\right) \overline{\frac{1}{\lambda-1}}+\Psi_{t}\right]-a \Psi_{t}\right)
\end{gathered}
$$

Si se elimina $\beta_{1}$ en la expresión anterior, se obtiene:

$$
\begin{gathered}
0=\left[\beta_{1}\left(1+b \beta_{0}\right)\right]^{\frac{1}{\lambda-1}} \frac{\left(W_{t}-\beta_{0} \Psi_{t}\right)^{\lambda}}{\lambda}-\rho \beta_{1} \frac{\left(W_{t}-\beta_{0} \Psi_{t}\right)^{\lambda}}{\lambda} \\
+\beta_{1}\left(W_{t}-\beta_{0} \Psi_{t}\right)^{\lambda-1} W_{t} r+\beta_{1}\left(W_{t}-\beta_{0} \Psi_{t}\right)^{\lambda-1} \\
W_{t}\left(\frac{\mu-r}{\lambda}\right)^{2} \frac{1}{(1-\lambda)^{2}}\left[1-\beta_{0} \frac{\Psi_{t}}{W_{t}}\right]^{2} \\
+\beta_{1}\left(W_{t}-\beta_{0} \Psi_{t}\right)^{\lambda-1} T_{t}-\beta_{1}\left(W_{t}-\beta_{0} \Psi_{t}\right)^{\lambda-1} \\
{\left[\Psi_{t}+\left[\beta_{1}\left(1+b \beta_{0}\right)\right]^{\frac{1}{\lambda-1}}\left(W_{t}-\beta_{0} \Psi_{t}\right)\right]} \\
+\frac{1}{2} \beta_{1}(\lambda-1)\left(W_{t}-\beta_{0} \Psi_{t}\right)^{\lambda-2} W_{t}^{2} \sigma^{2}\left[\frac{(\mu-r)}{\sigma_{t}^{2}(1-\lambda)} \frac{1}{W_{t}}\left(W_{t}-\beta_{0} \Psi_{t}\right)\right]^{2} \\
-\beta_{0} \beta_{1}\left(W_{t}-\beta_{0} \Psi_{t}\right)^{\lambda-1}\left(b\left[\beta_{1}\left(1+b \beta_{0}\right)\right]^{\frac{1}{\lambda-1}}\left[\left(W_{t}-\beta_{0} \Psi_{t}\right)+\Psi_{t}\right]-a \Psi_{t}\right)
\end{gathered}
$$

Se reducen términos iguales:

$$
\begin{aligned}
& 0=\left[\beta_{1}\left(1+b \beta_{0}\right)\right]^{\frac{1}{\lambda-1}} \frac{\left(a_{t}-\beta_{0} \Psi_{t}\right)^{\lambda}}{\lambda}-\rho \frac{\left(W_{t}-\beta_{0} \Psi_{t}\right)^{\lambda}}{\lambda} \\
& +\left(W_{t}-\beta_{0} \Psi_{t}\right)^{\lambda-1} W_{t} r+\frac{1}{2}\left(W_{t}-\beta_{0} \Psi_{t}\right)^{\lambda}\left(\frac{\mu-r}{\lambda}\right)^{2} \frac{1}{1-\lambda} \sigma^{2} \\
& +\left(W_{t}-\beta_{0} \Psi_{t}\right)^{\lambda-1} T_{t}-\left(W_{t}-\beta_{0} \Psi_{t}\right)^{\lambda}\left[\Psi_{t}+\left[\beta_{1}\left(1+b \beta_{0}\right)\right]^{\frac{1}{\lambda-1}}\right] \\
& -\beta_{0} \beta\left(W_{t}-\beta_{0} \Psi_{t}\right)^{\lambda-1}(a-b) \Psi_{t}-\left[\beta_{1}\left(1+b \beta_{0}\right)\right]^{\frac{1}{\lambda-1}}\left(W_{t}-\beta_{0} \Psi_{t}\right)^{\lambda}
\end{aligned}
$$


De esta manera,

$$
\begin{gathered}
0=\left[\beta_{1}\left(1+b \beta_{0}\right)\right]^{\frac{1}{\lambda-1}} \frac{\left(W_{t}-\beta_{0} \Psi_{t}\right)^{\lambda}}{\lambda}-\rho \frac{\left(W_{t}-\beta_{0} \Psi_{t}\right)^{\lambda}}{\lambda} \\
+\left(W_{t}-\beta_{0} \Psi_{t}\right)^{\lambda-1} W_{t} r+\frac{1}{2}\left(W_{t}-\beta_{0} \Psi_{t}\right)^{\lambda}\left(\frac{\mu-r}{\lambda}\right)^{2} \frac{1}{1-\lambda} \sigma^{2} \\
+\left(W_{t}-\beta_{0} \Psi_{t}\right)^{\lambda-1} T_{t}-\left(W_{t}-\beta_{0} \Psi_{t}\right)^{\lambda}\left[\Psi_{t}+\left[\beta_{1}\left(1+b \beta_{0}\right)\right]^{\frac{1}{\lambda-1}}\right] \\
-\beta_{0} \beta\left(W_{t}-\beta_{0} \Psi_{t}\right)^{\lambda-1}(a-b) \Psi_{t}-\left[\beta_{1}\left(1+b \beta_{0}\right)\right]^{\frac{1}{\lambda-1}}\left(W_{t}-\beta_{0} \Psi_{t}\right)^{\lambda}
\end{gathered}
$$

Si se considera que

$$
\Psi_{t}\left(1+\beta_{0}(b-a)\right)+W_{t} r=-r\left(W_{t}-1+\beta_{0}(b-a) \Psi_{t}\right),
$$

y que

o

$$
\beta_{0}=\frac{1+\beta_{0}(b-a)}{r}
$$

$$
\beta_{0}=\frac{1}{r+a-b}
$$

Por lo tanto se puede eliminar $\left(W_{t}-\beta_{0} x_{t}\right)^{\lambda}$ de todos los términos y se obtiene:

$$
\begin{gathered}
0=\frac{\left[\beta_{1}\left(1+b \frac{1}{r+a-b}\right)\right]^{\frac{1}{\lambda-1}}}{\lambda}-\frac{\rho}{\lambda}+\left(W_{t}-\frac{1}{r+a-b} \Psi_{t}\right)^{\lambda-1} \\
\left(W_{t} r-T_{t}-(a-b) \Psi_{t}\right)+\frac{1}{2}\left(\frac{\mu-r}{\lambda}\right)^{2} \frac{1}{1-\lambda} \sigma^{2}-\Psi_{t}
\end{gathered}
$$

En consecuencia,

$$
\begin{gathered}
\beta_{1}\left(1+b \frac{1}{r+a-b}\right) \\
=\left[\frac{\rho}{\lambda}+\left(W_{t} r-T_{t}-(a-b) \Psi_{t}\right)+\frac{1}{2}\left(\frac{\mu-r}{\lambda}\right)^{2} \frac{1}{1-\lambda} \sigma^{2}-\Psi_{t}\right]^{\lambda-1}
\end{gathered}
$$

Así,

$$
\beta_{1}=\left[\frac{1}{(1-\lambda)(r+a-b)-\lambda}\right]^{\lambda-1}
$$


Domínguez y Venegas, UN MODELO MICROECONÓMICO ESTOCÁSTICO

\section{Bibliografía}

Andrew, B. (1990), "Asset Prices under Habit Formation and Catching Up with the Joneses”, The American Economic Review, vol. 80, núm. 2, pp. 38-42.

Banxico (2010), Balanza de pagos ingreso por remesas 1990-2010, México, Banco de México <www.banxico.org.mx> (mayo de 2011).

Bodie, Z. y J. Detemple (2004), "Optimal Consumption-Portfolio Choices and Retirement Planning”, Journal of Economic Dynamic and Control, vol. 28, núm. 6, pp. 1115-1148.

Breeden, D. (1986), "Consumption, Production, Inflation and Interest Rates: A Synthesis”, Journal of Financial Economics, vol. 16, núm. 1, pp. 3-39.

Campbell, J. y J. Cochrane (1999), "By Force of Habit: A Consumption Based Explanation of Aggregate Stock Market Behavior”, The Journal of Political Economy, vol. 107, núm. 2, pp. 205-251.

Castillo, R.A. (2001), "Remesas un análisis de cointegración para el caso de México", Frontera Norte, vol. 13, núm. 26, pp. 31-50.

Chapman, A. (1998), "Habit Formation and Aggregate Consumption", Econometrica, vol. 66, núm. 5, pp. 1223-1230.

Conapo (2010), Índice de intensidad migratoria México-Estados Unidos, México, Consejo Nacional de Población <www.conapo.gob.mx> (junio de 2010>.

Constantinides, G. (1990), "Habit Formation: A Resolution of the Equity Premium Puzzle", Journal of Political Economy, vol. 98, núm. 3, pp. 519-543.

Cox, C. y C. Huang (1989), "Optimal Consumption and Portfolio Policies when Asset Prices Follow a Diffusion Process", Journal of Economic Theory, vol. 89, núm. 1, pp. 33-83.

Detemple, J. y F. Zapatero (1991), “Asset Prices in an Exchange Economy with Habit Formation”, Econometrica, vol. 59, núm. 6, pp. 1633-1657.

Gavirato, R. y A. Torres (2004), "Migración e impacto de las remesas en la economía nacional”, Análisis Económico, vol. 19, núm. 41, pp. 243-275.

Hall, R. (1978), "Stochastic Implications of the Life Cycle-Permanent Income Hypothesis: Theory and Evidence", Journal of Political Economic, vol. 86, núm. 6, pp. 971-987.

Hall, R. (1988), "Intertemporal Substitution in Consumption”, The Journal of Political Economy, vol. 96, núm. 2, pp. 339-357.

Hansen, L. y K. Singleton (1983), "Stochastic Consumption, Risk Aversion, and the Temporal Behavior of Asset Returns", Journal of Political Economy, vol. 91, núm. 2, pp. 249-265.

Heredia, C. (2011), "La migración mexicana y el debate en Estados Unidos", Nueva Sociedad, núm. 233, pp. 132-149.

Hey, J. y D. Valentino (1988), "Optimal Consumption under Uncertainty: An Experimental Investigation”, The Economic Journal, vol. 98, núm. 390, pp. 105-116.

Hicks, J. (1965), Capital and Growth, Nueva York, Oxford University Press. 
INEGI (2010), Censo de Población y Vivienda 2010, México, Instituto Nacional de Estadística y Geografía.

Lozano, F. (1998), "Las remesas de los migrantes mexicanos en Estados Unidos: estimaciones para 1995", "Migration between Mexico and United States", Binational Study, vol. 3, México, Secretaría de Relaciones Exteriores de México / U.S. Commission on Inmigration Reform.

Lozano, F. (1999), "Immigrants from Cities: New Trends in Urban-Origin Mexican Migration to the United States", tesis de doctorado, Austin, Universidad de Texas.

Mackinnon, James G., Alfred A. Haug y Leo Michelis (1999), "Numerical Distribution Functions of Likelihood Ratio Tests for Cointegration”, Journal of Applied Econometrics, vol. 14, núm. 5, pp. 563-577.

Márquez, C. (2006), "Consumo duradero, hábitos y mercados de valores", Revista Economía Aplicada, vol. 16, núm. 42, pp. 5-33.

Merton, R. (1969), "Lifetime Portfolio Selection under Uncertainty: The Continuous-Time", The Review of Economics and Statistics, vol. 51, núm. 3, pp. $247-257$.

Merton, R. (1971), "Optimal Consumption and Portfolio Rules in a Continuous Time Model”, Journal of Economic Theory, vol. 3, pp. 373-413.

Miller, B. (1974), "Optimal Consumption with a Stochastic Income Stream", Econometrica, vol. 42, núm. 2, pp. 253-266.

Pollak, S. (1970), "Habit Formation and Dynamic Demand Function”, Journal of Political Economy, vol. 78, núm. 4, pp. 745-763.

Ryder, H. y G. Heal (1973), "Optimal Growth with Intertemporally Dependent Preferences”, Review of Economic Studies, vol. 40, núm. 1, pp. 1-33.

Santiago, M.J. (2001), "Importancia económica de la migración internacional en México. Análisis desde la perspectiva de las remesas”, Momento Económico, núm. 114, pp. 41-47.

STPS (2012), Salario mínimo general de México 2012, México, Secretaría del Trabajo y Previsión Social <www.stps.gob.mx> (enero de 20012).

Sundaresan, S. (1989), "Intertemporally Dependent Preferences and the Volatility of Consumption and Wealth", Review of Financial Studies, vol. 2, núm.1, pp. 73-89.

Tuirán, R. (2006), "La migración mexicana hacia Estados Unidos: las reformas en puerta y retos futuros", Papeles de Población, núm. 48, pp. 9-31.

U.S. Department of Labor (2012), Census Bureau 2010, Washington, U.S. Department of Commerce, Economics and Statistics Administration <www. bls.gov> (mayo de 2012).

Zheng, X. y X. Xu (2003), "Optimal Portfolio Rules with Habit Formation and Preference for Wealth", Wuhan University Journal of Natural Sciences, vol. 8, núm. 4, pp. 1057-1060. 
Domínguez y Venegas, UN MODELO MICROECONÓMICO ESTOCÁSTICO

\section{Acerca de los autores}

Rosa María Domínguez Gijón es licenciada en Economía con especialidad en Economía de los Negocios por el Instituto Politécnico Nacional, y maestra en Economía con especialidad en Finanzas por la Escuela Superior de Economía del Instituto Politécnico Nacional (ESE-IPN). Es candidata a doctora en Ciencias Económicas en la ESE-IPN.

Francisco Venegas Martínez es doctor en Matemáticas y doctor en Economía por la Universidad Estatal de Washington, cuenta con un posdoctorado en Finanzas por la Universidad de Oxford y tiene estudios de doctorado en Física en la Universidad Autónoma Metropolitana. Obtuvo el Premio a la Investigación en el IPN en 2011, así como la Presea Lázaro Cárdenas 2012, otorgada a profesores e investigadores.

Su línea de investigación es economía financiera y se especializa en administración de riesgos financieros y matemáticas financieras. Actualmente es profesor de tiempo completo en el posgrado de la Escuela Superior de Economía del Instituto Politécnico Nacional. Pertenece al Sistema Nacional de Investigadores de Conacyt (SNI III). 
